Case Report

\title{
Small Bowel Adenocarcinoma as the Cause of Gastrointestinal Bleeding in Celiac Disease: A Rare Malignancy in a Common Disease
}

\author{
Jaleh Fallah,, ${ }^{1,2}$ Maxwell Eyram Afari, ${ }^{1,2}$ Alfredo C. Cordova, ${ }^{2,3}$ \\ Adam J. Olszewski, ${ }^{1,2}$ and Taro Minami ${ }^{2,4}$ \\ ${ }^{1}$ Department of Medicine, Memorial Hospital of Rhode Island, Pawtucket, RI 02860, USA \\ ${ }^{2}$ The Warren Alpert Medical School of Brown University, Pawtucket, RI 02860, USA \\ ${ }^{3}$ Department of Surgery, Memorial Hospital of Rhode Island, Pawtucket, RI 02860, USA \\ ${ }^{4}$ Division of Pulmonary, Critical Care and Sleep Medicine, Memorial Hospital of Rhode Island, Pawtucket, RI 02860, USA
}

Correspondence should be addressed to Jaleh Fallah; jfallah1191@gmail.com

Received 20 May 2015; Accepted 14 July 2015

Academic Editor: Yoshihito Yokoyama

Copyright (C) 2015 Jaleh Fallah et al. This is an open access article distributed under the Creative Commons Attribution License, which permits unrestricted use, distribution, and reproduction in any medium, provided the original work is properly cited.

\begin{abstract}
Introduction. Celiac disease is associated with an increased risk of small bowel malignancies, particularly lymphoma. Its association with small bowel carcinoma is less known. Case Description. We report a case of an 89-year-old woman with celiac disease who experienced recurrent episodes of gastrointestinal bleeding and was ultimately found to have adenocarcinoma of the small intestine. Discussion and Evaluation. Diagnosis of small bowel adenocarcinoma is often delayed because of the need for specialized modalities, which are often deferred in the inpatient setting. Although resection is the modality of choice for small bowel tumors, a majority is either locally advanced or metastatic at diagnosis, and even localized cancers have worse prognosis than stage-matched colorectal tumors. The role of adjuvant chemotherapy is uncertain, but it is often offered extrapolating data from other gastrointestinal cancers. Small bowel carcinomas occurring in the context of celiac disease appear to be associated with higher rates of microsatellite instability than sporadic tumors, although other specific genomic abnormalities and mechanisms of carcinogenesis in celiac disease remain unknown. Conclusion. Recurrent episodes of gastrointestinal bleeding in a patient with celiac disease should prompt an early evaluation of the small bowel to assure timely diagnosis of carcinoma at an early curable stage.
\end{abstract}

\section{Introduction}

Celiac disease is a common disease, with a prevalence of approximately $1-2 \%$ in North and South America, North Africa, India, and the Middle East [1]. Iron deficiency is a common clinical feature of this disease and is related to malabsorption rather than gastrointestinal blood loss. Celiac disease is known to be associated with an increased risk of malignancy, particularly lymphoma $[2,3]$. However, less is known about the risk of gastrointestinal carcinoma associated with celiac disease. We have encountered a case of adenocarcinoma of the small intestine with celiac disease, a rare malignancy in a common disease.

\section{Case Presentation}

An 89-year-old woman with a history of celiac disease, prior gastrointestinal bleeding, and dementia was admitted to our Intensive Care Unit for a recurrent gastrointestinal bleeding. The celiac disease had been diagnosed two years earlier by an endoscopic biopsy of the duodenum (demonstrating a subtotal villous atrophy) and with a correlative serum tissue transglutaminase IgA antibody (162 units, reference range 0-19 units). The patient had not been compliant with the recommended gluten-free diet because of early dementia and was treated with additional oral budesonide because of chronic abdominal discomfort. She was in her usual state 


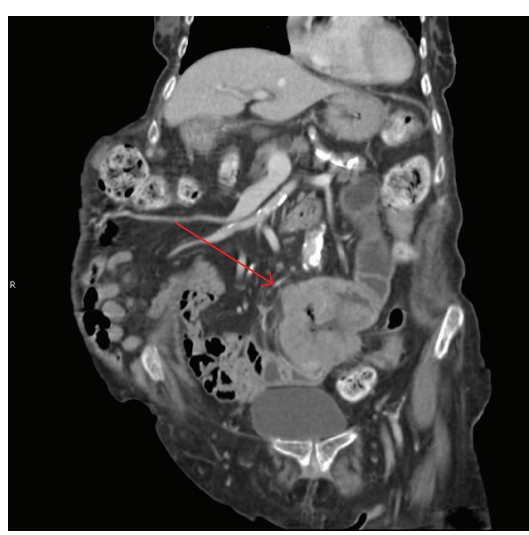

(a)

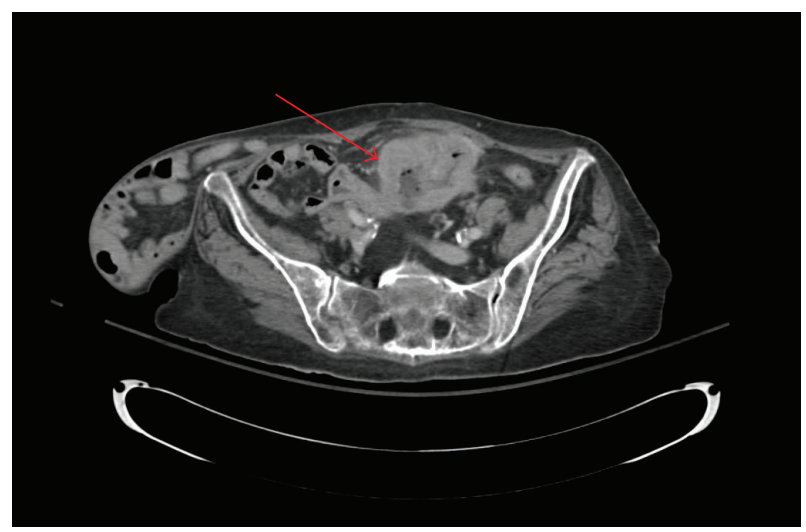

(b)

FIgURE 1: Axial (a) and coronal (b) projections of the computerized tomography of the abdomen. Arrows point to the small bowel tumor.

of health until two days prior to this admission when she developed nausea and had multiple bouts of coffee ground emesis, with which she presented to the emergency room. She reported poor appetite and significant weight loss during the prior year, which had been ascribed to celiac disease-related malabsorption and led to therapy with oral budesonide. Her medical history was additionally significant for prior admission with similar symptoms of nausea, vomiting, and gastrointestinal bleeding with severe anemia three, six, and twelve months prior to the index presentation. Esophagogastroduodenoscopy (EGD) had been performed on all these occasions, showing gastritis and duodenitis with biopsies consistent with celiac disease. There was no history of tobacco or alcohol use and no family history of celiac disease or malignancies.

On admission, the patient was hypotensive with blood pressure of $93 / 49 \mathrm{~mm} \mathrm{Hg}$ and heart rate of 86 beats per minute and afebrile and her respiratory rate was 18 breaths per minute. Physical examination revealed a palpable left paraumbilical mass and a $20 \mathrm{~cm} \times 20 \mathrm{~cm}$ reducible hernia in the right abdominal wall. Laboratory work-up revealed hemoglobin of $6.9 \mathrm{~g} / \mathrm{dL}$ (reference range, $12.0-16.0 \mathrm{~g} / \mathrm{dL}$ ). Serum chemistry, liver function tests, electrocardiogram, and chest radiograph were within normal limits. Abdominal computed tomography (CT) with intravenous contrast revealed a proximal dilatation of an abnormally thickened segment of the small bowel suspicious for a malignancy (Figure 1). The patient was resuscitated with intravenous fluids and a red cell transfusion.

On third admission day, after hemodynamic stability was established, she underwent laparotomy. A large $(15 \times 10 \mathrm{~cm})$ small bowel tumor located approximately $20 \mathrm{~cm}$ from the ligament of Treitz was resected (Figure 2). There was no evidence of metastatic lesions in the peritoneum or liver during intraoperative inspection of all quadrants of the abdominal cavity. Pathologic examination revealed a poorly differentiated adenocarcinoma (final tumor size $6.5 \mathrm{~cm}$ ) with invasion into an adjacent portion of the small intestine and to the serosal surface, with metastases to 4 out of 12 resected lymph nodes, staged as pT4b pN2 M0 or stage III according

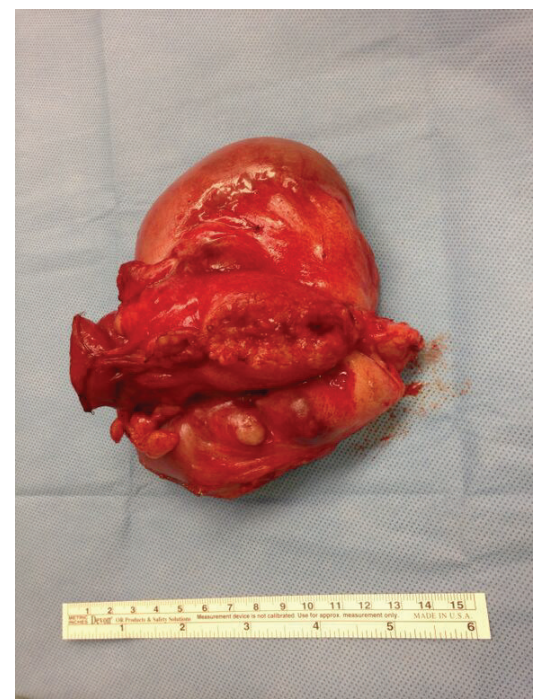

FIGURE 2: Photograph of the gross resection specimen of the small bowel segment containing adenocarcinoma.

to the American Joint Commission on Cancer staging system (Figure 3). The tumor cells were positive for immunohistochemical markers of adenocarcinoma (CDX2 and CK7) and negative for CK20, vimentin, neuroendocrine markers (synaptophysin, chromogranin, and CD56), and lymphoid antigens (CD3, CD20, and CD45). After the surgery, the patient elected palliative care and did not receive any further cancer-directed therapy.

\section{Discussion}

Small bowel carcinoma is a rare clinical entity with an annual incidence of 0.66 cases per 100,000 person-years in the United States, which is higher for men $(0.80$ per 100,000$)$ than for women (0.55) [4]. Neuroendocrine tumors (39\%, mostly carcinoids) are more common than carcinomas (31\%), while less common histologies encompass lymphomas (18\%) and 


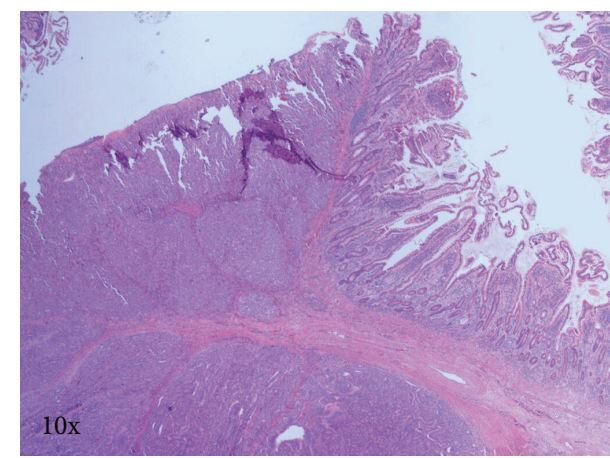

(a)

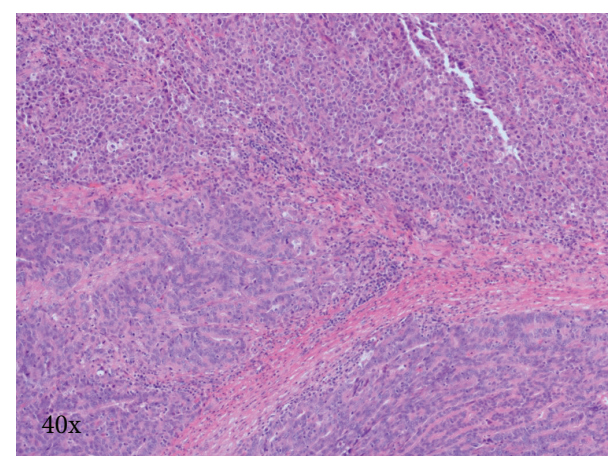

(b)

FIgURE 3: Microscopic images of the tumor from the histopathologic specimen, hematoxylin and eosin stain. (a) Low-power magnification (10x) shows normal small bowel mucosa (right) and invasive adenocarcinoma (left). (b) High-power magnification (40x) shows two patterns of differentiation, with poorly differentiated carcinoma in the upper part of the panel.

sarcomas (10\%). Although the small intestine is three times longer than the large intestine, it is the primary location for only $5 \%$ of all gastrointestinal malignancies [5].

Despite the fact that lower gastrointestinal bleeding is a common cause of hospitalization in the elderly, the diagnosis can be challenging and the diagnostic differential is broad. In a study of 1,112 patients admitted to an urban emergency medical center, diverticulosis (33.5\%), hemorrhoids (22.5\%), and carcinoma (12.7\%) were the most common etiologies [6]. In general the causes of gastrointestinal bleeding can be grouped into anatomic (diverticular disease), vascular (hemorrhoid, ischemic colitis, and angiodysplasia), neoplastic, and inflammatory (infectious colitis, inflammatory bowel disease) causes.

The diagnosis of small bowel carcinoma is difficult and often delayed. Clinical presentation is typically nonspecific with symptoms that include nausea, vomiting, weight loss, abdominal pain, and anemia. In our patient, the diagnostic process was further complicated by presence of a known small bowel disease (celiac disease) commonly associated with iron deficiency anemia, negative endoscopy, and colonoscopy. Iron deficiency anemia is present in $46 \%$ of patients with celiac disease and is typically caused by malabsorption of iron in the proximal small intestine and usually resolves with strict adherence to gluten-free diet and oral iron supplementation, although it may be refractory in up to $20 \%$ [7]. In our case, the correct diagnosis for the underlying cause of recurrent anemia was only made during the fourth hospital admission.

Serum cancer markers carcinoembryonic antigen (CEA) and Ca19.9 may be elevated in cases of small bowel carcinoma but are neither sensitive nor specific and should not be used for diagnostic purposes. Even in metastatic disease CEA is elevated only in $30 \%$ and Ca19.9 in $41 \%$ of cases [8]. Extrapolating the established guidelines for colorectal carcinoma, these markers, if elevated at diagnosis, can be used for surveillance after surgery or for assessment of disease activity in patients with metastatic cancer [9]. Values of CEA or Ca19.9 were not obtained in our patient who elected palliative care only after her cancer diagnosis.
Because small bowel is not accessible during standard endoscopic procedures performed in the setting of gastrointestinal bleeding, a high index of suspicion is required to identify pathology in this segment of the gastrointestinal tract. Clinical examination has a low sensitivity for small bowel tumors until they become palpable, as it was in our case. CT may provide suggestive information about nodal, extramucosal involvement and distant metastases. However, it can miss superficial lesions and presence of overlapping bowel loops may lead to false negative diagnosis. The addition of enterography to CT circumvents these problems and increases the sensitivity [10]. Magnetic resonance imaging (MRI) is rapidly gaining clinical utility in the evaluation of small bowel disease. It advantageously avoids ionizing radiation, can assess bowel peristalsis, and provides a higher resolution, but it has its own challenges such as cost and accessibility. CT is better in the assessment of a perforation or bowel obstruction and concomitantly helps in directing interventional therapies [10].

Capsule endoscopy may identify a site of occult bleeding or malignancy but poses a risk of capsule retention when there is an impending obstruction [11]. Double balloon enteroscopy overcomes the above limitation and has the advantage of ability to obtain biopsy or providing endoscopic therapy [12]. These newer diagnostic techniques can expedite the diagnosis, but in clinical practice they are often deferred to the outpatient setting in patients presenting with acute gastrointestinal bleeding. Our case illustrates the importance of pursuing such evaluations when repeated episodes of unexplained gastrointestinal bleeding occur in order to avoid delay in the diagnosis of a potentially curable malignancy.

The pathogenesis of small bowel adenocarcinoma is not entirely known due to the rarity of the disease. Genetic syndromes like Peutz-Jeghers (relative risk, 520), hereditary nonpolyposis colon cancer (relative risk, 103 to 291 depending on specific mutation), and familial adenomatous polyposis (relative risk, 330) are risk factors for small bowel adenocarcinoma [13]. Conditions associated with chronic inflammation of the small intestine are also major risk factors for this malignancy and include Crohn's disease and celiac disease. 
Thirteen percent of small bowel adenocarcinomas have been shown to be associated with celiac disease [14]. Cases of small bowel adenocarcinoma in patients with celiac disease have been previously reported [15-17].

Celiac disease is an autoimmune disorder which results from the interplay of immune, genetic, and environmental factors. The lymphocytic infiltrate could potentially induce an immunological response disrupting the epithelium and causing premalignant changes. In a series of 94 cases of small bowel adenocarcinoma from the Mayo Clinic, evidence of flat dysplasia at the tumor margin was noted in $50 \%$ of cases associated with celiac disease [18]. Although some authors suggested that carcinomas in celiac disease may arise from adenomatous polyp [19], others found no increased incidence of duodenal adenomas in celiac disease [20].

Microsatellite instability, a hallmark of the hereditary nonpolyposis colon cancer (Lynch) syndrome, is a molecular feature commonly encountered in small bowel adenocarcinoma, where it is present in about $20-40 \%$ of cases [21-23]. Several studies have demonstrated particularly high prevalence of microsatellite instability (70-80\%) in tumors associated with celiac disease $[24,25]$. A comparative genomic hybridization study of 15 celiac disease-related and 33 unrelated small bowel adenocarcinomas showed similar chromosomal aberrations between the two subsets, but there was a higher rate of $A P C$ gene promoter hypermethylation and of microsatellite instability in celiac disease-related tumors (67\% versus 33\%) [26].

Small bowel carcinogenesis in Crohn's disease differs from celiac disease. Cytokines, released due to the chronic inflammatory state, interact with cell surface receptors and target genes thus promoting carcinogenesis [27]. Unlike in celiac disease where proximal jejunum predominates as the location of adenocarcinomas, Crohn's disease affects mostly the distal ileum.

In the absence of randomized trials, the optimal treatment for small bowel carcinoma is still uncertain and is essentially extrapolated from the clinical experience in colorectal cancer. Surgery remains the treatment of choice in the absence of metastatic disease and is often required even in the metastatic setting due to high likelihood of obstruction or severe hemorrhage [28]. Because most patients are diagnosed with advanced disease and have high-grade histology [4], small bowel adenocarcinoma generally carries a poor prognosis with 5-year relative survival of $28 \%$ without surgery and 32\% after resection [29]. Adjuvant chemotherapy using fluorouracil-based regimens approved in colorectal cancer is commonly offered for patients with stage II or III disease [30]. It may also provide benefits in selected patients with metastatic disease, where a $27 \%$ response rate, median progression-free survival of 6.6 months and median overall survival of 15 months, was reported among patients receiving palliative chemotherapy [8]. In one phase II trial of the combination of capecitabine and oxaliplatin, a response rate of $50 \%$ was observed, with median time to progression of 11.3 months and median overall survival of 20.4 months [31]. It is important however to recognize that patients with significant comorbidities or poor performance status may not derive any benefit from adjuvant or palliative chemotherapy, and, in those cases, as it was in ours, supportive care alone may be appropriate.

Identification of molecular pathways involved in the pathogenesis of small bowel cancer holds a promise of developing more efficacious therapies. A screening assay of 140 compounds suggested utility of the halichondrin B analogue eribulin in cases with Wnt- $\beta$-catenin pathway mutation present in $20 \%$ of cases [21]. A multiplex assay analysis of 83 cases showed frequent mutations in KRAS (43\%), TP53 (41\%), PIK3CA (8\%), BRAF (6\%), and ERBB2 (8\%) genes, for which targeted agents are available or currently studied [32].

\section{Conclusion}

When patients with celiac disease present with gastrointestinal bleeding, small intestinal malignancy has to be included in differential diagnosis if endoscopy and colonoscopy do not reveal an obvious source of blood loss. Early evaluation for a malignancy using a high-yield technique should be considered to avoid a diagnostic delay and the poor prognosis associated with advanced cancers.

\section{Abbreviations}

CT: Computerized tomography

EGD: Esophagogastroduodenoscopy.

\section{Consent}

Written informed consent for the publication of this paper could not be obtained due to patient death. All patient identifiers have been removed to ensure patient's privacy.

\section{Conflict of Interests}

The authors declare no conflict of interests.

\section{Authors' Contribution}

All authors were involved in the formulation and drafting of the case report. All authors have read and approved the final paper. Alfredo C. Cordova was the surgeon operating on the patient.

\section{Acknowledgment}

The authors extend their sincere gratitude to Dr. Stanley Schwartz for the preparation and review of pathology.

\section{References}

[1] N. E. Castillo, T. G. Theethira, and D. A. Leffler, "The present and the future in the diagnosis and management of celiac disease," Gastroenterology Report, vol. 3, no. 1, pp. 3-11, 2015.

[2] C. Catassi, E. Fabiani, G. Corrao et al., "Risk of non-Hodgkin lymphoma in celiac disease," Journal of the American Medical Association, vol. 287, no. 11, pp. 1413-1419, 2002.

[3] J. F. Ludvigsson, S. M. Montgomery, A. Ekbom, L. Brandt, and F. Granath, "Small-intestinal histopathology and mortality risk 
in celiac disease," Journal of the American Medical Association, vol. 302, no. 11, pp. 1171-1178, 2009.

[4] O. Qubaiah, S. S. Devesa, C. E. Platz, M. M. Huycke, and G. M. Dores, "Small intestinal cancer: a population-based study of incidence and survival patterns in the United States, 1992 to 2006," Cancer Epidemiology Biomarkers and Prevention, vol. 19, no. 8, pp. 1908-1918, 2010.

[5] A. I. Neugut, J. S. Jacobson, S. Suh, R. Mukherjee, and N. Arber, "The epidemiology of cancer of the small bowel," Cancer Epidemiology, Biomarkers \& Prevention, vol. 7, no. 3, pp. 243251, 1998.

[6] C. Gayer, A. Chino, C. Lucas et al., "Acute lower gastrointestinal bleeding in 1,112 patients admitted to an urban emergency medical center," Surgery, vol. 146, no. 4, pp. 600-607, 2009.

[7] T. R. Halfdanarson, M. R. Litzow, and J. A. Murray, "Hematologic manifestations of celiac disease," Blood, vol. 109, no. 2, pp. 412-421, 2007.

[8] A. Zaanan, L. Costes, M. Gauthier et al., "Chemotherapy of advanced small-bowel adenocarcinoma: a multicenter AGEO study," Annals of Oncology, vol. 21, no. 9, pp. 1786-1793, 2010.

[9] G. Y. Locker, S. Hamilton, J. Harris et al., "ASCO 2006 update of recommendations for the use of tumor markers in gastrointestinal cancer," Journal of Clinical Oncology, vol. 24, no. 33, pp. 5313-5327, 2006.

[10] G. Masselli and G. Gualdi, "CT and MR enterography in evaluating small bowel diseases: when to use which modality?" Abdominal Imaging, vol. 38, no. 2, pp. 249-259, 2013.

[11] R. Srai, L. Tullie, A. Wadoodi, and M. Saunders, "Capsule endoscopy: a dangerous but diagnostic tool," BMJ Case Reports, 2013.

[12] H. Suzuki, A. Yamada, H. Watabe et al., "Successful treatment of early-stage jejunum adenocarcinoma by endoscopic mucosal resection using double-balloon endoscopy: a case report," Diagnostic and Therapeutic Endoscopy, vol. 2012, Article ID 521960, 4 pages, 2012.

[13] T. Aparicio, A. Zaanan, M. Svrcek et al., "Small bowel adenocarcinoma: epidemiology, risk factors, diagnosis and treatment," Digestive and Liver Disease, vol. 46, no. 2, pp. 97-104, 2014.

[14] P. D. Howdle, P. K. Jalal, G. K. T. Holmes, and R. S. Houlston, "Primary small-bowel malignancy in the UK and its association with coeliac disease," QJM, vol. 96, no. 5, pp. 345-353, 2003.

[15] H. Benhammane, F. Z. El M'rabet, K. Idrissi Serhouchni et al., "Small bowel adenocarcinoma complicating coeliac disease: a report of three cases and the literature review," Case Reports in Oncological Medicine, vol. 2012, Article ID 935183, 4 pages, 2012.

[16] M. Gwiggner and P. Patel, "An unusual case of obscure gastrointestinal bleeding in a patient with coeliac disease," Case Reports in Gastrointestinal Medicine, vol. 2011, Article ID 634684, 3 pages, 2011.

[17] S. D. Rampertab, K. A. Forde, and P. H. R. Green, "Small bowel neoplasia in coeliac disease," Gut, vol. 52, no. 8, pp. 1211-1214, 2003.

[18] C. J. Bruno, K. P. Batts, and D. A. Ahlquist, "Evidence against flat dysplasia as a regional field defect in small bowel adenocarcinoma associated with celiac sprue," Mayo Clinic Proceedings, vol. 72, no. 4, pp. 320-322, 1997.

[19] C. Catassi, I. Bearzi, and G. K. T. Holmes, "Association of celiac disease and intestinal lymphomas and other cancers," Gastroenterology, vol. 128, no. 4, pp. S79-S86, 2005.
[20] S. D. Rampertab, A. Fleischauer, A. I. Neugut, and P. H. R. Green, "Risk of duodenal adenoma in celiac disease," Scandinavian Journal of Gastroenterology, vol. 38, no. 8, pp. 831-833, 2003.

[21] H. Suzuki, Y. Hirata, N. Suzuki et al., "Characterization of a new small bowel adenocarcinoma cell line and screening of anti-cancer drug against small bowel adenocarcinoma," The American Journal of Pathology, vol. 185, no. 2, pp. 550-562, 2015.

[22] M. Planck, K. Ericson, Z. Piotrowska, B. Halvarsson, E. Rambech, and M. Nilbert, "Microsatellite instability and expression of MLH1 and MSH2 in carcinomas of the small intestine," Cancer, vol. 97, no. 6, pp. 1551-1557, 2003.

[23] T. Aparicio, M. Svrcek, A. Zaanan et al., "Small bowel adenocarcinoma phenotyping, a clinicobiological prognostic study," British Journal of Cancer, vol. 109, no. 12, pp. 3057-3066, 2013.

[24] D. D. Potter, J. A. Murray, J. H. Donohue et al., "The role of defective mismatch repair in small bowel adenocarcinoma in celiac disease," Cancer Research, vol. 64, no. 19, pp. 7073-7077, 2004.

[25] F. Bergmann, S. Singh, S. Michel et al., "Small bowel adenocarcinomas in celiac disease follow the CIM-MSI pathway," Oncology Reports, vol. 24, no. 6, pp. 1535-1539, 2010.

[26] B. Diosdado, T. E. Buffart, R. Watkins et al., "High-resolution array comparative genomic hybridization in sporadic and celiac disease-related small bowel adenocarcinomas," Clinical Cancer Research, vol. 16, no. 5, pp. 1391-1401, 2010.

[27] V. Palascak-Juif, A. M. Bouvier, J. Cosnes et al., "Small bowel adenocarcinoma in patients with Crohn's disease compared with small bowel adenocarcinoma de novo," Inflammatory Bowel Diseases, vol. 11, no. 9, pp. 828-832, 2005.

[28] M. S. Talamonti, L. H. Goetz, S. Rao, and R. J. Joehl, "Primary cancers of the small bowel: analysis of prognostic factors and results of surgical management," Archives of Surgery, vol. 137, no. 5, pp. 564-571, 2002.

[29] S. Y. Pan and H. Morrison, "Epidemiology of cancer of the small intestine," World Journal of Gastrointestinal Oncology, vol. 3, no. 3, pp. 33-42, 2011.

[30] M. J. Overman, S. Kopetz, E. Lin, J. L. Abbruzzese, and R. A. Wolff, "Is there a role for adjuvant therapy in resected adenocarcinoma of the small intestine," Acta Oncologica, vol. 49, no. 4, pp. 474-479, 2010.

[31] M. J. Overman, G. R. Varadhachary, S. Kopetz et al., "Phase II study of capecitabine and oxaliplatin for advanced adenocarcinoma of the small bowel and ampulla of vater," Journal of Clinical Oncology, vol. 27, no. 16, pp. 2598-2603, 2009.

[32] A. Laforest, T. Aparicio, A. Zaanan et al., "ERBB2 gene as a potential therapeutic target in small bowel adenocarcinoma," European Journal of Cancer, vol. 50, no. 10, pp. 1740-1746, 2014. 


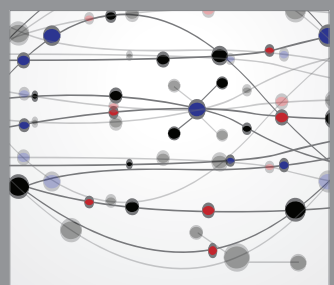

The Scientific World Journal
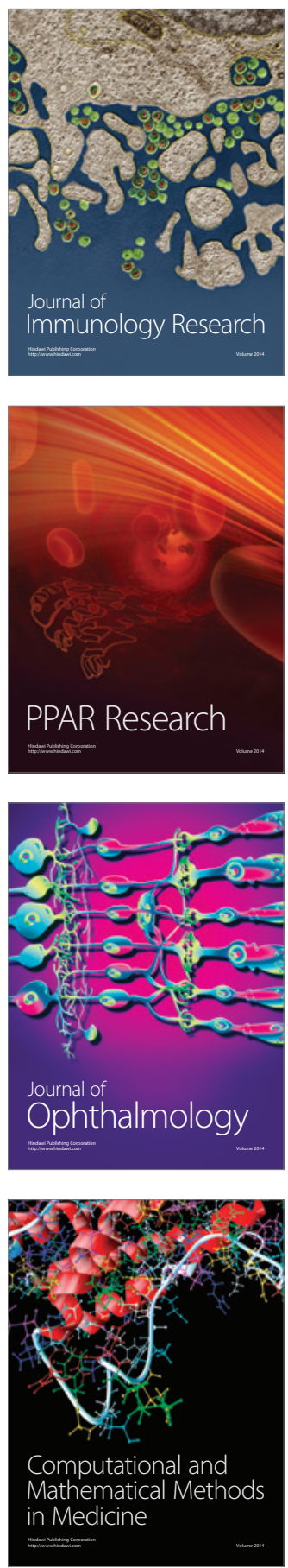

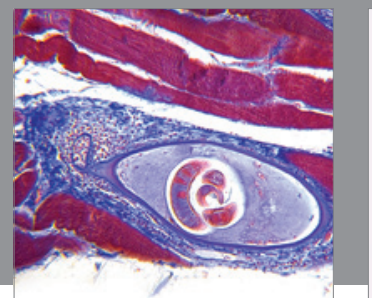

Gastroenterology

Research and Practice
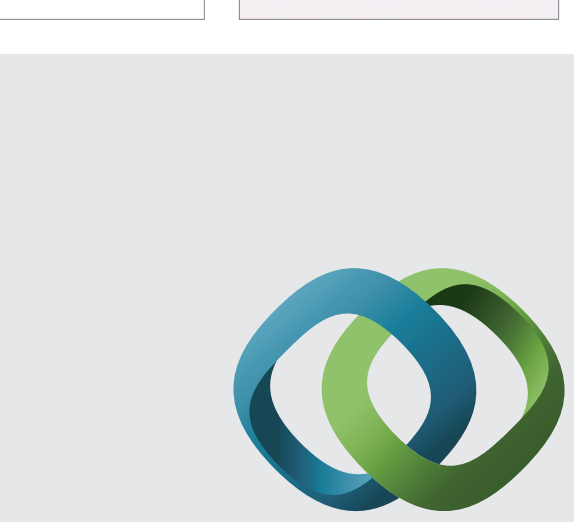

\section{Hindawi}

Submit your manuscripts at

http://www.hindawi.com
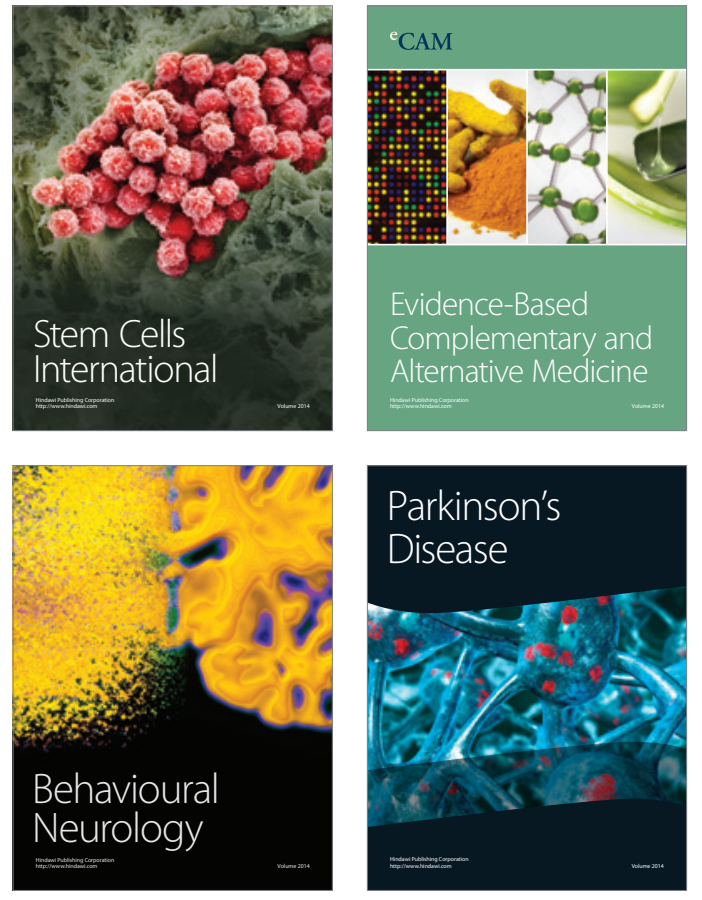
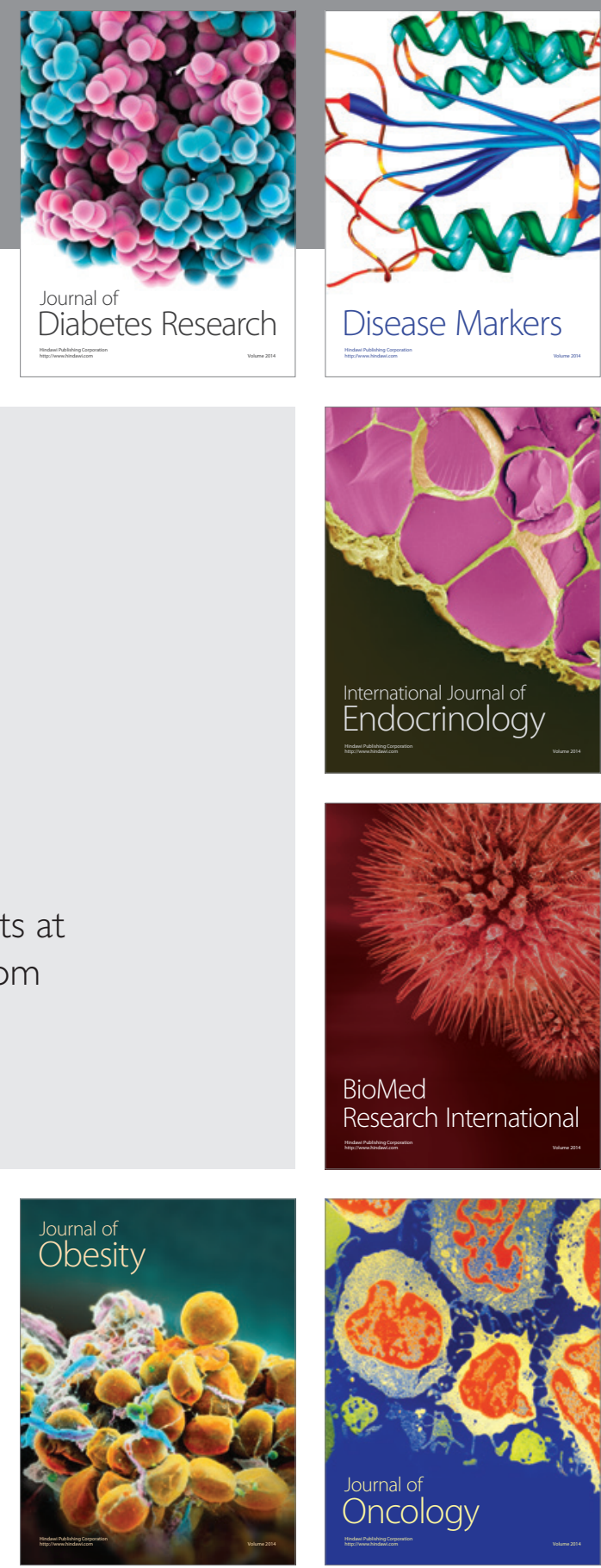

Disease Markers
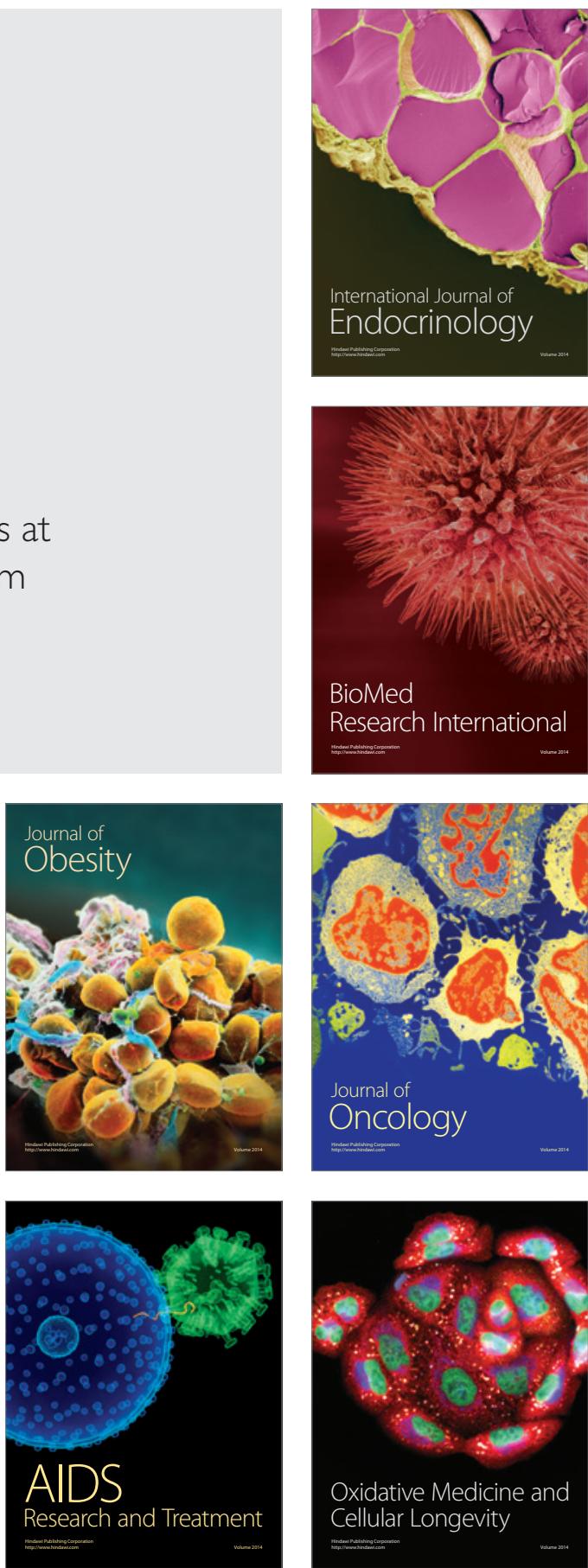